reason for the pain in the groin may be that the tendinitis was in the trochanteric region, but that the quadratus femoris originates from the tuberosity of the ischium. It could be a referred pain.

To the authors' knowledge quadratus femoris tendinitis as a cause of groin pain has not been reported in the medical literature before. There are several publications on groin pain, especially affecting athletes. ${ }^{1-5}$ Inguinal hernia (sports hernia), tendinitis (adductor tendons, rectus femoris tendon, rectus abdominis tendon, iliopsoas tendon), bursitis, osteitis, symphysitis, and prostatitis have been described as causes of groin pain.

From this case we concluded that when physical examination and herniography do not point to the "ordinary" causes of groin pain, quadratus femoris tendinitis should be considered. Magnetic resonance imaging can confirm the diagnosis and may avoid delay in the appropiate treatment, which may be conservative with ultrasound guided local corticosteroids injections.

1 Ashby EC. Chronic obscure pain is commonly caused by enthesopathy: "tennis elbow" of the groin. $\mathrm{Br} \mathcal{F}$ Surg 1994;81:1632-4.

2 Ekberg O, Persson NH, Abrahanson P-A, Westlin NE, Lilja B. Longstanding groin pain in athletes. Sports Med 1988;6: $56-61$.

3 Karlsson J, Swärd L, Kälebo P, Thomée R. Chronic groin injuries in athletes. Sports Med 1994;17:141-8.

4 Renström PAFH. Tendon and muscular injuries in the groin area. Clin Sports Med 1992;11:815-31.

5 Zimmerman G. Groin pain in athletes. Aust Fam Physician 1988;17:1046-52.

\title{
Local complications of self administered anabolic steroid injections
}

\author{
Nick A Evans
}

\begin{abstract}
In addition to the pharmacological side effects of anabolic steroids, complications may also result from the injection technique used in self administration. Two cases are presented where anabolic steroid injections resulted in knee joint sepsis and radial nerve palsy.

(Br f Sports Med 1997;31:349-350)
\end{abstract}

Keywords: anabolic steroids; injections; complications

Intramuscular injection is a popular route of self administering anabolic steroids to improve athletic performance or improve body image. Ninety six per cent of steroid users in a recent study admitted to using injectable preparations. ${ }^{1}$ This report highlights two unusual clinical complications resulting from anabolic steroid injections, where the athletes initially chose to withhold the relevant illicit drug history.

\section{Case reports}

CASE 1

Department of

Trauma and

Orthopaedics,

Morriston Hospital,

Swansea SA9 6NL,

United Kingdom

N A Evans

Correspondence to Mr N A Evans, Department of Orthopaedics, East Glamorgan Hospital, Pontypridd CF38 1AB.
On further questioning, the athlete admitted to self administering intramuscular injections of anabolic steroid (stanozolol $50 \mathrm{mg} / \mathrm{ml}$, Zam- bon, Spain) directly into the vastus medialis in an attempt to specifically cause an increase in its size. Three separate injections of $1 \mathrm{ml}$ were administered into this area during the previous week, and although sterile needles were used, he did not seem to observe aseptic technique.

The knee joint was lavaged arthroscopically, and a small area of synovial inflammation was noted in the medial aspect of the suprapatellar pouch, presumably the site of misplaced intramuscular injections. On culture the knee joint fluid grew Staphylococcus aureus. The patient did not have any predisposition to staphylococcal infection, and made an uneventful recovery with supplemental antibiotics.

\section{CASE 2}

A 28 year old recreational bodybuilder presented to his general practitioner with a short history of parasthesia over the dorsum of his left hand. A small area of changed sensation was identified together with mild weakness (MRC grade 4) of left wrist extension. The patient linked the symptoms to a left sided neck sprain sustained while lifting weights one week earlier. With a suspicion of cervical nerve root compression, specialist opinion was sought.

When the patient was seen in clinic one month later, his neurological symptoms had resolved spontaneously. Clinical examination failed to identify any neurological deficit in the left upper limb, neck movements were full and pain free. However, skeletal muscle hypertrophy was noted, along with the traid of physical signs associated with anabolic steroid use, namely acne, gynaecomastia and striae. ${ }^{1}$ When confronted, he admitted to steroid use, and in particular, injecting nandrolone decanoate 
(Organon, UK) directly into his left triceps, near the origin of the lateral head, immediately before the onset of his neurological symptoms. The diagnosis of injection injury to the radial nerve, thus became apparent.

\section{Discussion}

The pharmacological side effects of anabolic steroids are well known, but complications may also arise from the injection technique used in self administration. Poor injection technique has resulted in inadvertent sciatic nerve injury, ${ }^{2}$ and cross infection with HIV has been reported after needle sharing. ${ }^{34}$ The cases presented here show complications resulting from the use of unconventional injection sites.

Sterile syringes and needles can be obtained from needle exchange schemes, or from black market suppliers. Those users attending needle exchange schemes may be educated on techniques of intramuscular injection, ${ }^{2}$ but others learn such technical details from more experienced drug users, or from steroid manuals. Nevertheless, the principles of asepsis may not be adhered to, particularly when injections are administered in unsavoury environments such as the gymnasium changing room, by a training partner or coach. It is also doubtful whether counterfeit black market products are subject to the appropriate sterilisation processes.

Other potential problems arise in relation to the anatomical location of the intramuscular injection. The steroid regimens used by veteren users and competitive bodybuilders require frequent, often daily injections. ${ }^{1}$ Thus, to provide respite for the more traditional injecting areas of the gluteal region, deltoid and vastus lateralis, users will inject in unconventional muscle sites. Furthermore, some bodybuilders believe that injecting directly into specific muscles will locally increase muscle size. Anecdotally, a lagging, smaller muscle group can be selectively injected with anabolic steroid to boost its size. Thus despite any attempts of appropriate tuition, intramuscular injections may often resemble a "lucky dip", where needles are inserted without any knowledge of the underlying anatomy and regional neurovascular network.

In the above case reports, the bodybuilders used unconventional injection sites for both these reasons. Case 1 chose to inject vastus medialis to induce a localised anabolic effect, unaware of the location of the suprapatellar pouch, and inadvertently injected the steroid directly into the knee joint, which resulted in a septic arthritis. In case 2 , the athlete injected into the triceps because his glutei and deltoid muscles were painful from previous injections. The temporary radial nerve symptoms may have been the result of direct needle trauma or local nerve compression from the injected bolus.

These cases illustrate two important messages regarding unsupervised anabolic steroid use. Firstly, steroid users may inject into unconventional muscle sites, which can result in unusual clinical complications. Secondly, athletes may not be forthcoming with the drug history, either because of the illicit nature of the drug use, or because there is a genuine ignorance that the symptoms are a direct result of poor injection technique. In these situations, identifying typical physical signs of anabolic steroid use, ${ }^{1}$ namely acne, striae and gynaecomastia, may provide a clue in solving a potential clinical mystery.

Evans NA. Gym and tonic: a profile of 100 male steroid users. Br $\mathcal{f}$ Sports Med 1997;31:54-8.

2 Perry HM. An unusual cause of abnormal gait. $B r \mathcal{Y}$ Sports Med 1994;28:60.

3 Sklarek HM, Mantovani RP, Erens E, Heisler D, Niederman MS, Fein AM. AIDS in a bodybuilder using anabolic steroids. N Engl ₹ Med 1984;311:1701.

4 Scott MJ, Scott MJ Jr. HIV infection associated with injections of anabolic steroids. $f A M A 1989 ; 262: 207-8$.

5 Phillips WN. Anabolic reference guide. Golden, Colorado: Mile High Publishing, 1991. 